\title{
NOUVELLE
}

\section{Rôle du gène MBD4 dans un phénotype hypermutateur et dans la tumorigenèse}

Manuel Rodrigues ${ }^{1,2}$, Lenha Mobuchon ${ }^{1}$, Alexandre Houy ${ }^{1}$, Anne-Céline Derrien ${ }^{1}$, Alice Fiévet ${ }^{3}$, Marc-Henri Stern ${ }^{1,3}$
${ }^{1}$ Institut Curie, Université de recherche PSL, Inserm U830, DNA repair and uveal melanoma (D.R.U.M.), Équipe labellisée par la Ligue nationale contre le cancer, Paris, 75248, France

${ }^{2}$ Département d'oncologie médicale, Institut Curie, Université de recherche PSL, Paris, 75248, France

${ }^{3}$ Département de génétique, Institut Curie, Université de recherche PSL, Paris, 75248, France

marc-henri.stern@curie.fr
> Le mélanome uvéal (MU) est une forme rare de mélanome d'origine oculaire, représentant environ

$5 \%$ de l'ensemble $\rightarrow$ Voir la Synthèse des mélanomes [1] $m / s n^{\circ} 2$, février 2018 $(\rightarrow)$. Contrairement page 155

aux mélanomes cutanés, les MU sont caractérisés par l'une des plus faibles charges mutationnelles parmi toutes les tumeurs solides de l'adulte et par l'absence de signature mutationnelle liée aux rayonnements ultra-violets. Cependant, certains MU ne suivent pas ces règles générales et, quoiqu'exceptionnels, ces cas sont souvent informatifs.

Nous avons récemment rapporté le cas d'un patient traité par un anticorps dirigé contre un point de contrôle immunitaire (PDl [programmed cell death 1]) pour un MU métastatique qui a présenté une réponse tumorale exceptionnelle à cette drogue (Figure 1) [2]. Le taux de réponse à ces thérapies anti-points de contrôle immunitaire est de l'ordre de $5 \%$ dans cette maladie [3], probablement limité par la très faible charge mutationnelle et, en conséquence, le faible nombre de néo-épitopes. Les échantillons de la tumeur primaire comme des métastases de ce patient répondeur présentaient une très importante charge mutationnelle de près de 300 mutations somatiques par exome, soit environ 20 fois plus importante que celle habituellement observée dans les MU (Figure 2A). Le répertoire des mutations dans une tumeur reflète les processus mutationnels endogènes et exogènes auxquels les cellules pro-

génitrices ont été exposées de la vie embryonnaire jusqu'à la formation de la tumeur. Plus de $90 \%$ des mutations ponctuelles observées dans cette tumeur répondant à l'immunothérapie étaient des transitions cytosine en thymine dans un contexte de cytosine suivie d'une guanine $(\mathrm{CpG}>\mathrm{TpG}$ ) (Figure 2B). Ce phénotype était très évocateur d'un processus de désamination des cytosines méthylées. La désamination des 5-méthylcytosines est un événement mutationnel spontané générant des mutations somatiques $C p G>T p G$ à un taux constant. Par conséquent, le niveau de ces mutations dans une tumeur est dans la plupart des cancers corrélé à l'âge de l'individu au moment du diagnostic [4]. Les désaminations des cytosines sont habituellement efficacement prises en charge par un mécanisme de réparation par excision de bases, dont la première étape est le clivage de la base altérée par une glycosylase: TDG (thymine DNA glycosylase) ou MBD4 (methyl-

Figure 1. Un patient avec un mélanome uvéal métastatique présentant une réponse majeure à l'immunothérapie. A. Histoire clinique du patient. B. Réponse complète sur la métastase hépatique (indiquée par une flèche). L'étoile indique un kyste hépatique sans rapport avec la tumeur. Le Pembrolizumab est un anticorps dirigé contre un point de contrôle immunitaire (anti-PDl [programmed cell death 1]). 


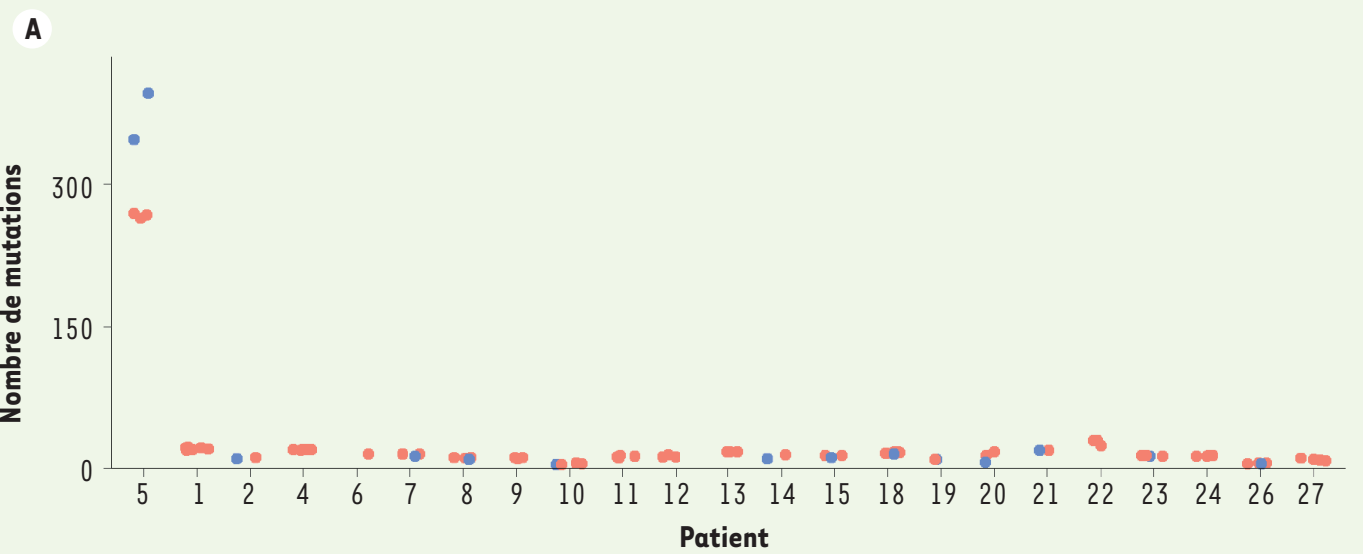

- Primaire - Métastase

B

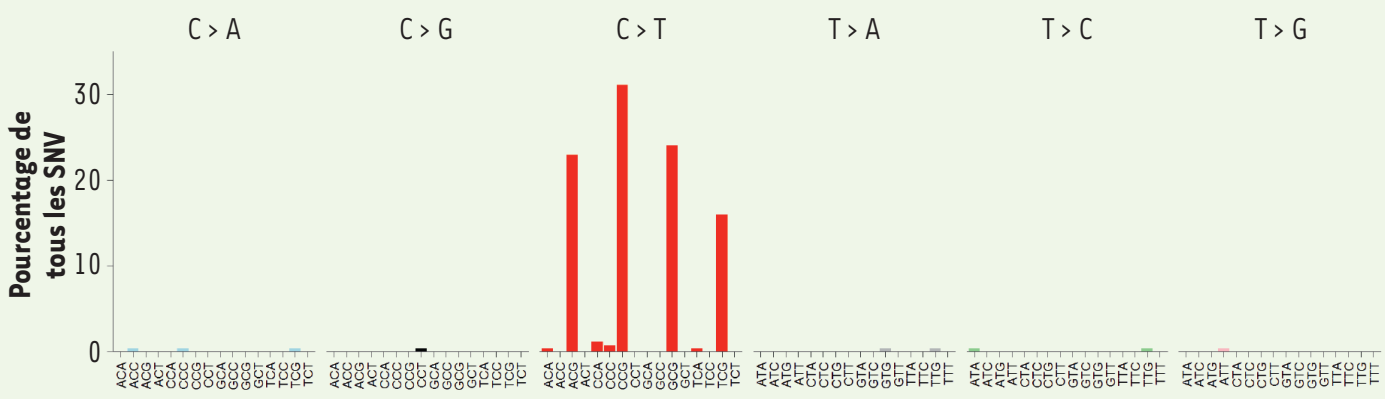

Figure 2. Phénotype mutateur du patient répondeur. A. Charge mutationnelle (nombre de mutations par exome et par échantillon tumoral) du patient répondeur $\left(n^{\circ} 5\right)$ par rapport aux autres patients présentant un mélanome uvéal métastatique (plusieurs échantillons par patient peuvent être analysés). B. Profil mutationnel de la tumeur répondeuse. Le pourcentage des différentes mutations possibles est indiqué selon le contexte nucléotidique (nucléotide avant et après la mutation). SNV : single nucleotide variant.

binding domain 4) [5]. MBD4 serait spécifique des lésions induites par la désamination des méthyl-CpG, alors que TDG est une enzyme plus polyvalente capable de traiter l'ensemble des lésions de désamination. Nous avons identifié chez ce patient une délétion constitutionnelle délétère d'une base de la séquence codante de MBD4 ([c.144ldel], p.[Leu482Trpfs*9] : délétion de la base 1441 et mutation de la leucine 482 en tryptophane, suivie d'un changement de cadre de lecture et d'un codon stop après 9 bases [fs: frameshift]), associée à une perte de l'allèle sauvage dans la tumeur résultant en une inactivation complète de ce gène. II s'agissait de la seule mutation de MBD4 retrouvée dans une série de MU dont les autres tumeurs n'étaient pas hypermutées. L'exploration systématique des données publiques disponibles pour les 10 000 tumeurs du programme «the cancer genome atlas (TCGA) » a permis d'identifier deux autres tumeurs - un autre MU et un glioblastome - avec le même phénotype hypermutateur $\mathrm{CpG}$ $>\mathrm{TpG}$, toutes deux porteuses de mutations constitutionnelles délétères de MBD4 avec perte de l'allèle sauvage dans la tumeur.

Le rôle de MBD4 dans la réparation de I'ADN est connu depuis une vingtaine d'années $[6,7]$. Dans les modèles murins, l'inactivation de Mbd4 induit une multiplication par 3 du nombre de transitions somatiques $\mathrm{CpG}>\operatorname{TpG}[8$, 9]. Chez l'homme, des mutations tumorales délétères de MBD4 ont été observées dans des séries limitées de cancers colorectaux et gastriques présentant un déficit de réparation des mésappariements (mismatch repair; MMR). Cependant, l'inactivation constitutionnelle de Mbd4 chez les souris déficientes pour MMR ne modifiait pas significativement le délai d'apparition des tumeurs ni le spectre tumoral [10]. Ces données suggèrent que des mutations de MBD4 sont susceptibles d'être induites par un déficit du MMR, mais les conséquences d'une telle inactivation, dans un contexte d'hypermutations, ne sont pas évidentes. Notre observation est 
la première identification d'un phénomène hypermutateur $C p G>T p G$ induit par la mutation constitutionnelle de MBD4 chez l'homme. Les trois mutations constitutionnelles identifiées sont retrouvées dans la population générale à de très faibles fréquences $\left(<10^{-4}\right.$ dans la base $\varepsilon_{x A C^{1}}$ ). Ceci suggère que ces mutations sont un facteur de prédisposition aux MU et aux glioblastomes, les trois patients identifiés n'avaient toutefois ni un âge au diagnostic remarquablement jeune, ni d'autres tumeurs associées. De plus, aucune augmentation de l'incidence de tumeurs n'a été observée dans les modèles murins qui présentent une inactivation de Mbd4. Cependant, des modèles doublement inactivés pour Mbd4 et pour le gène Apc (adenomatous polyposis coli) présentaient une survie réduite, un doublement de la masse tumorale et un taux de mutations supérieur aux souris seulement déficientes en Apc. L'inactivation de MBD4 dans la lignée germinale pourrait être insuffisante pour initier la tumorigenèse, mais augmenterait la charge mutationnelle dans les tumeurs. Sa contribution à la progression tumorale dans les tumeurs humaines reste encore mal comprise.

La découverte d'un nouveau processus mutagène dans l'une des tumeurs solides de l'adulte avec la plus faible charge mutationnelle peut sembler contre-intuitive. Une des raisons envi-

${ }^{1}$ ExAC est un consortium qui a l'objectif d'agréger et d'harmoniser des données de séquençage d'exomes. http://exac. broadinstitute.org/ sageables serait que la perte récurrente du chromosome 3 , sur lequel se trouve MBD4, favoriserait l'inactivation complète de ce gène dans les MU. La monosomie 3 dans les MU est systématiquement associée à une inactivation de BAPI (BRCAl [breast cancer type 1] associated protein 1), gène majeur du remodelage de l'épigénome qui code une ubiquitine carboxyl-terminal hydrolase. Cependant, la perte partielle ou complète du chromosome 3 est un évènement récurrent dans un grand nombre de tumeurs solides de l'adulte et ne peut donc à elle seule expliquer l'inactivation de MBD4 dans les MU. Le cas du glioblastome exprimant MBD4 muté que nous rapportons présentait une inactivation d'ATRX (X-linked helicase II). Une publication récente décrit également trois cas de leucémies aiguës myéloblastiques mutées pour MBD4 qui présentent des mutations de gènes remodeleurs de l'épigénome tels que $I D H I / 2$ [isocitrate déshydroxygénase 1 ou 2] et DNMT3A [DNA methyltransferase 3A]) [11]. Les conséquences de l'inactivation de MBD4 sur le remaniement de l'épigénome et sur la tumorigenèse représentent ainsi des pistes majeures à explorer dans les thérapies anti-cancéreuses. $\diamond$

Role of MBD4 in hypermutator phenotype and malignant transformation

\section{REMERCIEMENTS}

Ce travail a été financé par la Commission européenne (programme H2020 UMCURE n 667787), la cancéropôle lle de-France, l'INCa, I'Inserm, la Ligue nationale contre le cancer (Équipe label- lisée) et l'Institut Curie. MR a été financé par le programme doctoral «Formation à la recherche translationelle» de l'INCa/ITMO/AVIESAN PhD et LM par H2020.

\section{LIENS D'INTÉRÊT}

Les auteurs déclarent n'avoir aucun lien d'intérêt concernant les données publiées dans cet article.

\section{RÉFÉRENCES}

1. Alsafadi S, Mobuchon L, Rodrigues M, Stern M-H. Le mélanome uvéal- Une maladie modèle des anomalies de l'épissage et de l'oncogenèse. Med Sci (Paris) 2018 ; 34 : 155-60.

2. Rodrigues M, Mobuchon L, Houy A, et al. Outlier response to anti-PDl in uveal melanoma reveals germline MBD4 mutations in hypermutated tumors. Nat Commun 2018 ; 9 : 1866.

3. Algazi AP, Tsai KK, Shoushtari AN, et al. Clinical outcomes in metastatic uveal melanoma treated with PD-1 and PD-Ll antibodies. Cancer 2016 ; 122 : 334453.

4. Alexandrov LB, Jones PH, Wedge DC, et al. Clock-like mutational processes in human somatic cells. Nat Genet $2015 ; 47: 1402-7$.

5. Sjolund AB, Senejani AG, Sweasy JB. MBD4 and TDG: multifaceted DNA glycosylases with ever expanding biological roles. Mutat Res 2013 ; 743-744 : 12-25.

6. Bellacosa A, Cicchillitti L, Schepis F, et al. MED1, a novel human methyl-CpG-binding endonuclease, interacts with DNA mismatch repair protein MLHI. Proc Natl Acad Sci U S A 1999 ; 96 : 3969-74.

7. Hendrich $B$, Hardeland $\mathrm{U}, \mathrm{Ng} \mathrm{HH}$, et al. The thymine glycosylase MBD4 can bind to the product of deamination at methylated $\mathrm{CpG}$ sites. Nature 1999 ; 401 : 301-4.

8. Millar CB, Guy J, Sansom 0J, et al. Enhanced CpG mutability and tumorigenesis in MBD4-deficient mice. Science $2002 ; 297$ : 403-5.

9. Wong $\varepsilon$, Yang K, Kuraguchi M, et al. Mbd4 inactivation increases Cright-arrowT transition mutations and promotes gastrointestinal tumor formation. Proc Natl Acad Sci U S A 2002 ; 99 : 14937-42.

10. Tricarico R, Cortellino S, Riccio A, et al. Involvement of MBD4 inactivation in mismatch repair-deficient tumorigenesis. Oncotarget $2015 ; 6: 42892-904$.

11. Sanders MA, Chew $\varepsilon$, Flensburg C et al. MBD4 guards against methylation damage and germline deficiency predisposes to clonal hematopoiesis and early-onset AML. Blood 2018 ; pii: blood-2018-05-852566. doi: 10.1182/blood-2018-05-852566

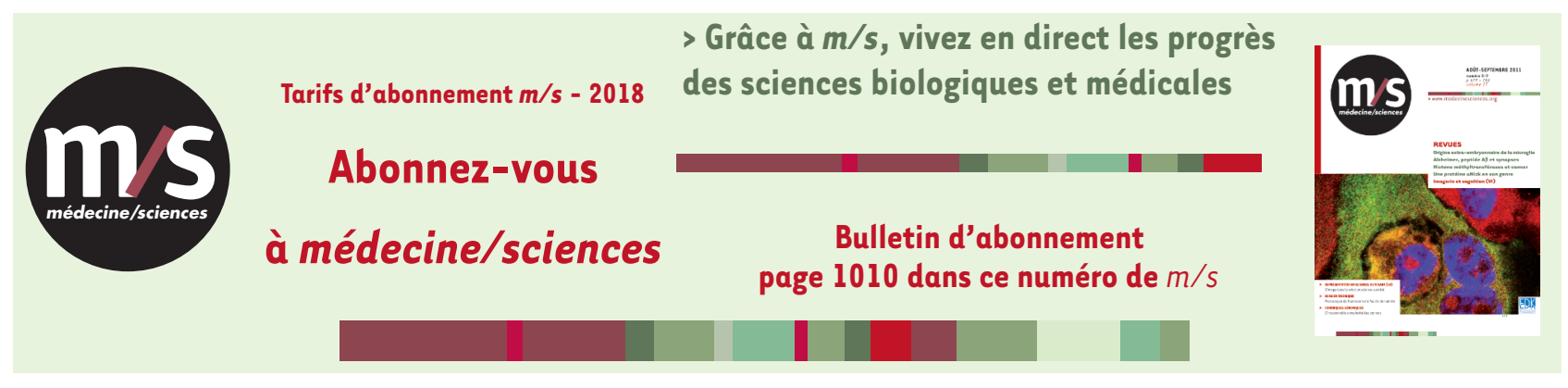

